\title{
Stochastic geometric properties of scalar interfaces in turbulent jets
}

\author{
Paul L. Miller and Paul E. Dimotakis \\ Graduate Aeronautical Laboratories, California Institute of Technology, Pasadena, California 91125
}

(Received 26 July 1989; accepted 14 September 1990)

\begin{abstract}
Experiments were conducted in which the behavior of scalar interfaces in turbulent jets was examined, using laser-induced fluorescence (LIF) techniques. The experiments were carried out in a high Schmidt number fluid (water), on the jet centerline, over a jet Reynolds number range of $1000 \leqslant \operatorname{Re} \leqslant 24000$. Both two-dimensional scalar data, $c(r, t)$ at fixed $x / d$, and onedimensional scalar data, $c(t)$ at fixed $x / d$ and $r / x$, were analyzed using standard one- and twodimensional fractal box-counting algorithms. Careful treatment was given to the handling of noise. Both long and short records as well as off-centerline measurements were also investigated. The important effect of threshold upon the results is discussed. No evidence was found of a constant (power-law) fractal dimension over the range of Reynolds numbers studied. On the other hand, the results are consistent with the computed behavior of a simple stochastic model of interface geometry.
\end{abstract}

\section{INTRODUCTION}

The proposals of Mandelbrot ${ }^{1-4}$ to account for the stochastic geometry of turbulent interfaces in terms of powerlaw fractal similarity generated considerable hope in the turbulence community. The proposed formalism held the promise of an alternate interpretation of a variety of important quantities in turbulence related to energy spectra and dissipation as well as a description of the behavior of the interfacial surface of scalars and mixing down to molecular diffusion scales (e.g., Gouldin ${ }^{5}$ and Sreenivasan et al. ${ }^{6}$ ). The availability of scalar concentration and image data from several turbulent flows in our laboratory led us to a search for a power-law fractal description, i.e,, a similarity scaling wherein the number of elements $N(\lambda)$, of an extent $\lambda$, required to cover the scalar interface would be given by

$$
N(\lambda) \propto \lambda^{-D} \text {. }
$$

In the work presented here, we report on the results of our investigations on the behavior of scalar interfaces in turbulent jets using laser induced fuorescence (LIF) techniques and a fractal box-counting algorithm to analyze the data. In the time since this work was begun, results have appeared in the literature of experimental measures of fractals in turbulent shear flows by several investigations. See, for example, Sreenivasan and Meneveau, ${ }^{7}$ Sreenivasan et $a l .{ }^{6}$ and Prasad and Sreenivasan. ${ }^{8.9}$ They report that, in a variety of turbulent flows, including turbulent jets, scalar isoconcentration surfaces exhibit a constant fractal dimension in a small neighborhood of about $D=2.36$.

\section{APPARATUS AND PROCEDURE}

The experiments were carried out utilizing the facility shown in Fig. 1. A large rectangular water tank of about two cubic meters volume acted as the (discharge) reservoir. Large windows on all sides of the tank provided optical access. To establish the flow, the jet plenum was filled with water tagged by a fluorescent laser dye (sodium fiuorescein), and air was sonically metered to drive the jet fluid at constant velocity through a $2.54 \mathrm{~mm}(0.1 \mathrm{in}$.) diam nozzle at the base of the plenum. The bearn from an argon ion laser was passed through appropriate optics and aligned radially through the centerline of the jet. A beam stop prevented reffections from the opposite window. In the two-dimensional measurements, a line segment centered on the jet axis, at $x / d=300$, was imaged onto a linear photodiode array. The array was then scanned during a run, providing streak image data of concentration in one spatial dimension versus time (cf. Dimotakis et al. ${ }^{10}$ Dahm, ${ }^{11}$ Green and Losi, ${ }^{12}$ and Dahm and Dimotakis ${ }^{13}$ ).

For the single-point measurements, the laser beam optics were designed to generate a small Gaussian waist at the focus. A low laser power of $1.0 \mathrm{~W}$ was used to avoid heating of the dyed fluid in the very small focal volume and to prevent saturation. The plenum dye concentration was kept less than $10^{-6} \mathrm{M}$. Consequently, the much lower concentrations at the measuring station did not significantly attenuate or steer the beam. The resulting signal to noise was limited primarily by the 12-bit dynamic range of the $A / D$ converter, rather than shot noise from the low fiuorescence intensity.

The single-point measurements were made utilizing a low $f$ \# lens to collect light from a very short segment centered at the waist of the focused laser beam (on the jet centerline, at $x / d=100$ ) onto a photomultiplier tube, yielding single-point concentration values versus time. An optical low-pass filter eliminated background laser light, passing only the frequency-shifted fluorescence. A slit spatial filter defined the length of the laser line segment sampled. The slit width was chosen such that the sampling volume was roughly cubic in shape, about $80 \mu \mathrm{m}$ on each side. The use of the slit rather than a pinhole ensured that any small beam movetments in the vertical direction did not alter the measurement volume. The long Rayleigh range and latitude in the depth of field minimized the effect of motion in either of the two horizontal directions. Data acquisition was computer controlled. The signal amplifier incorporated a three-pole Butterworth filter, with a cutoff frequency set slightly under 10 $\mathrm{kHz}$. The data were sampled at $20 \mathrm{kHz}$ for all the runs. 


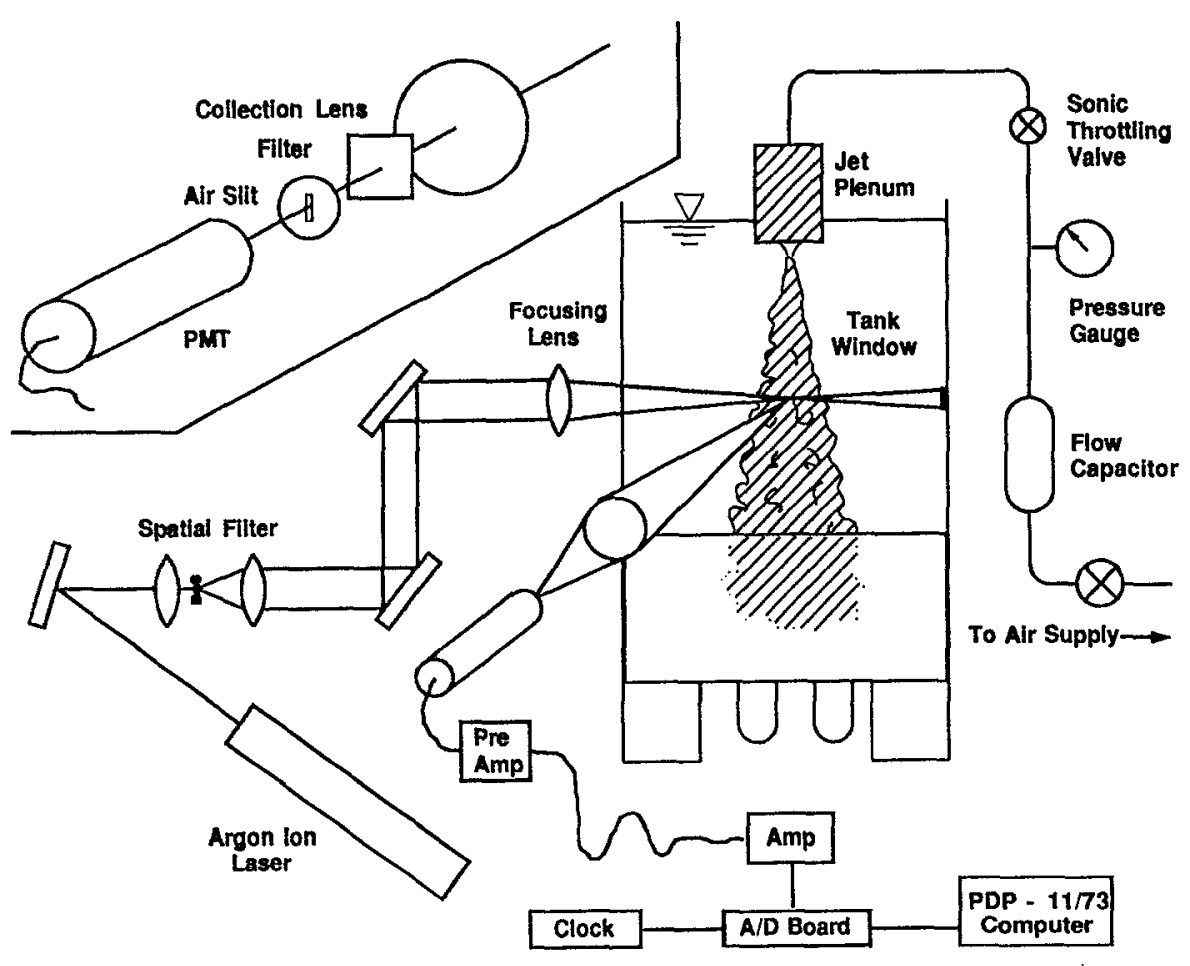

FIG. 1. Experimental apparatus.

\section{RESULTS AND DISCUSSION}

The single-point scalar concentration measurements were made as a function of time on the jet centerline, for a range of Reynolds numbers from 2940 to 23400 . The Reynolds number used here is defined as

$$
\operatorname{Re}=u_{0} d / v,
$$

where $u_{0}$ is the jet nozzle velocity, $d$ is the jet nozzle diameter, and $v$ is the kinematic viscosity. Careful consideration was given to the treatment of noise. Specifically, power spectra of the data were calculated, allowing the optimal (leastmean-squared error) Wiener filter (Wiener, ${ }^{14}$ Press et al., ${ }^{15}$ Dowling, ${ }^{16}$ and Dowling et al. ${ }^{17}$ ) to be obtained. The data were then convolved with the Wiener kernel to obtain the optimally estimated signal, consistent with the detection noise level in each run. Sample concentration power spectra, $S_{c}(f)$, before and after the filtering process, are compared in Fig. 2. The normalization time $\tau_{\mathrm{LS}}$ is the large scale passage time, $\delta / U_{c}$, where $\delta$ is the local visual jet diameter and $U_{c}$ is the mean centerline velocity calculated from the decay law reported by Chen and Rodi ${ }^{18}$ (cf. Dowling ${ }^{16}$ ).

In order to apply the Wiener filter effectively, the data were intentionally oversampled. This is clearly seen by the extent of the noise floor in the unfiltered spectrum of Fig. 2. Note that the signal-to-noise ratio (SNR) is a function of frequency, since the total power spectrum increases with decreasing frequency, while the noise is well described as white, or of constant level. We recognize that there are not only requirements of spatial and temporal resolution on such measurements, but also what we could call "SNR resolution," located roughly at the intersection of the signal spectrum and the noise floor. In the single-point measurements discussed here, spatial resolution was typically the most restrictive of the three.
The Wiener-filtered data were subsequently thresholded and transitions, or crossings of the threshold value, were located. The threshold value chosen was the local mean concentration $\bar{c}$. This is an unambiguous choice: it is close to both the mode of the concentration PDF and the value where the most transitions are obtained. From the vantage point of scalar diffusion, $\bar{c}$ is also the value toward which the local scalar field is driven by the diffusion process (scalar dissipation). The effect of the choice of alternate thresholds on the results is, however, an important issue and is discussed below.

The resulting record of transition locations was then processed using a one-dimensional fractal box-counting algorithm. The box-counting algorithm determines the num-

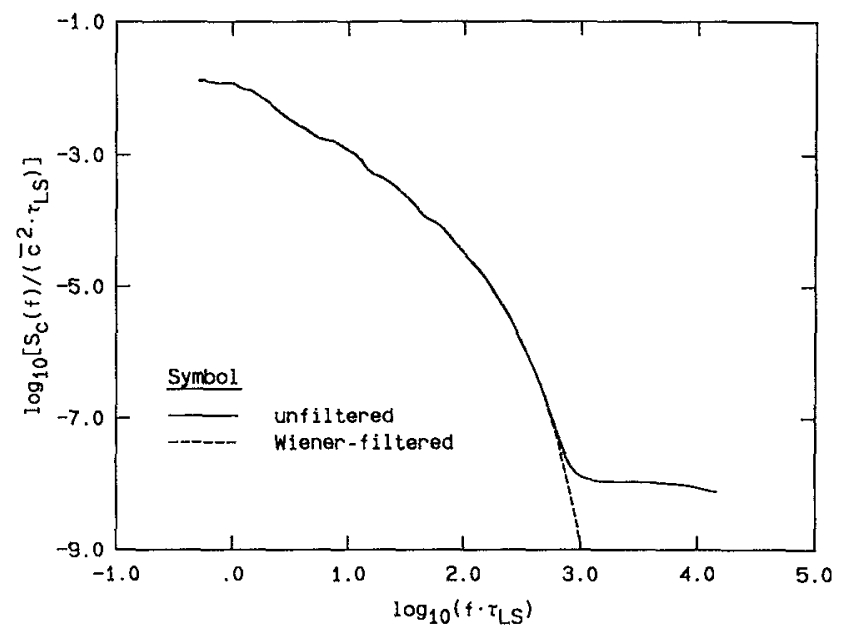

FIG. 2. Demonstration of the Wiener filter. 
ber $N$ of "tiles" (contiguous, constant length segments) required to cover the transition locations on a record, as a function of the tile size $\lambda$. The logarithm of $N(\lambda)$ may then be plotted as a function of $\log (\lambda)$. The logarithmic derivative,

$$
D(\lambda) \equiv-d \log [N(\lambda)] / d \log (\lambda),
$$

which is equal to minus the slope of the curve on this log-log plot, is then interpreted as the associated (local scale) fractal dimension.

As a demonstration of the box-counting algorithm utilized, a record was generated numerically of a Cantor set (with a necessarily finite range of scales) and processed. The result of the calculation is included in Fig. 3. The calculated points are connected with straight lines in the plots to aid the eye. The constant slope region is clearly visible, although there is some fluctuation about the expected analytic value of 0.631 . This may correspond to the oscillations observed by Smith et al. ${ }^{19}$ in numerical calculations of the fractal dimension for this set. The deviation at the smallest values of $\lambda$ is attributable to the lack of smaller features in this representation of the Cantor set. At these smallest scales, transitions occupy an entire box on the line containing the set, while in a true Cantor set, the transitions have zero measure. Thus there are fewer transitions than there ought to be and this reduces $D(\lambda)$ for small $\lambda$. At the largest scales, with $\lambda$ approaching the finite record length, there are no gaps between transitions of the size of the record or larger. As a result, large gaps are under-represented and $D(\lambda)$ rises. The deviations from the power-law fractal similarity behavior at the two extremes are therefore a consequence of the finite range of scales represented in the record and not an artifact of the processing.

The $\log -\log$ plots of $N(\lambda)$ and plots of the resulting $D(\lambda)$ for our high resolution, single-point on-axis concentration measurements in the jet, using a threshold value equal to the mean concentration, are shown in Figs. 4 and 5. The length scale, estimated from the calculated mean (centerline) velocity, has been expressed in absolute length (meters).

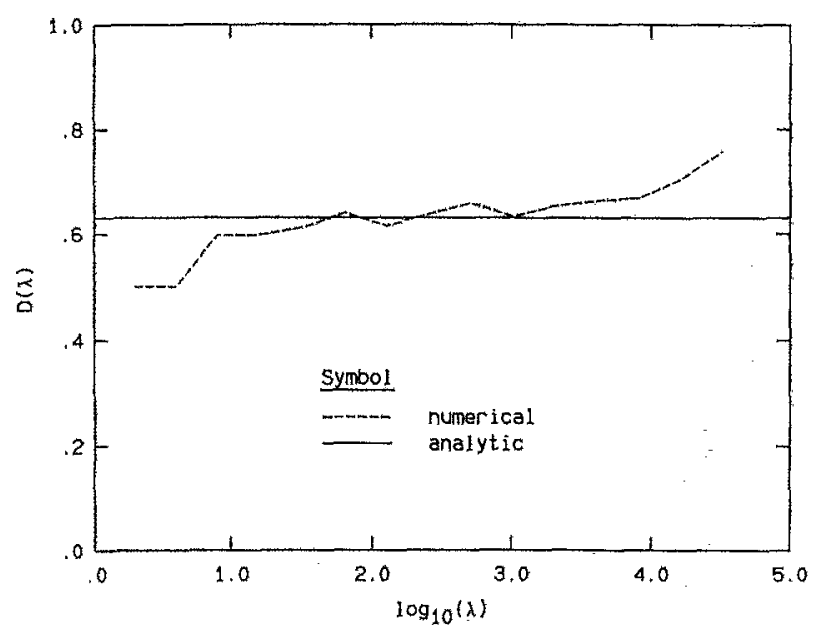

FIG. 3. Calculation for the Cantor set.

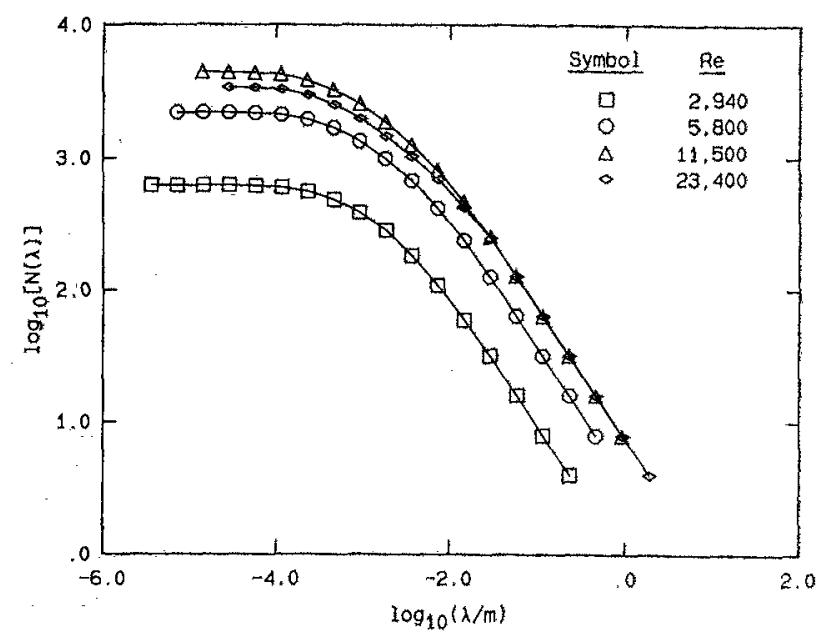

FIG. 4. Log-log tiling plots of centerline data for four Reynolds numbers $(x / d=100)$. Note that $\log _{10}(\delta / m) \approx-1.0$.

If the data were characterized by a constant (powerlaw) fractal behavior, one would observe a horizontal region on the curves in Fig. 5. As can be seen, there is no evidence of a constant value on the $D(\lambda)$ plots, other than the limiting values of 0 and 1 . It is for this reason that we denoted the logarithmic derivative of the $N(\lambda)$ curve by $D(\lambda)$ [Eq. (3) ], rather than simply $D$. The observed smooth variation in $D(\lambda)$ occurs over a range of equivalent spatial scales from below the Kolmogorov scale (see the discussion below), up to the outer large scales of the flow. The limiting value of 1 at the large tile sizes indicates that every tile of sufficient length covers transitions. This is to be expected for scales on the order of the jet diameter (about $11 \mathrm{~cm}$ for the data in Fig. 5), or larger, since over such a distance (or corresponding time) a crossing of the mean concentration level is almost certain. Failure to reach an asymptotic value of unity would indicate that either the data record was of insufficient length to capture the largest scales of the flow, or that the processing

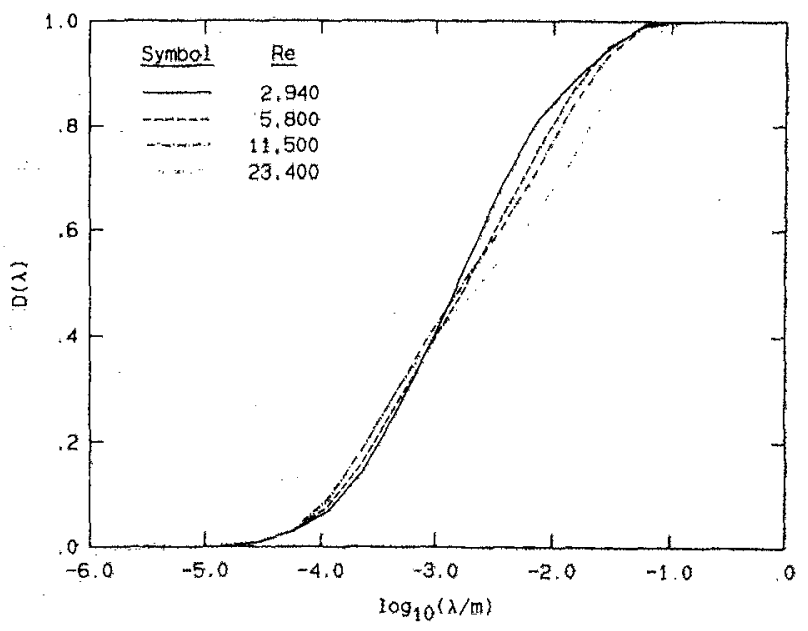

FIG. 5. Corresponding $D(\lambda)$ plots for Fig. 4. Here again $\log _{10}(\delta / m)$ $\approx-1.0$. For small scales, see the text. 
algorithm stopped at a tile size shorter than the largest scales.

Note that all four curves in Fig. 5 merge in the vicinity of $80 \mu \mathrm{m}$. This is consistent with our estimate of the spatial resolution of these measurements. To see how this relates to the spatial resolution requirements for this flow, we can estimate the Kolmogorov ${ }^{20}$ scale directly from its definition in terms of the mean energy dissipation rate, i.e.,

$$
\lambda_{\mathrm{K}} \equiv\left(v^{3} / \epsilon\right)^{1 / 4} \text {. }
$$

Using the result of Friehe $e t a l^{21}$ for the dissipation rate $\epsilon$ on the centerline of a turbulent jet (cf. Dowling ${ }^{16}$ ), i.e.,

$$
\epsilon=48\left(u_{0}^{3} / d\right)\left(\frac{\left(x-x_{0}\right)}{d}\right)^{-4},
$$

the Kolmogorov scales for these measurements are found to range from roughly 50 to $250 \mu \mathrm{m}$. By similarity arguments, the velocity field spatial scale where the action of viscosity will become important, say $\lambda_{v}$, will be some multiple of $\lambda_{\mathrm{K}}$. Normalized energy spectra are found to break from a constant power law at a wave number $k_{v}$ such that $k_{v} \lambda_{\mathrm{K}} \approx \frac{1}{8}$ (e.g., Chapman ${ }^{22}$ ). This yields an estimate of

$$
\lambda_{v}=\pi / k_{v} \sim 25 \lambda_{\mathrm{K}} \text {. }
$$

The smallest expected scalar diffusion (Batchelor ${ }^{23}$ ) scale $\lambda_{s}$ is smaller yet by a factor of $\mathrm{Sc}^{1 / 2}$, or $25-30$ in this case, yielding an estimate for $\lambda_{2}$ very close to $\lambda_{\mathrm{K}}$ (in water). These estimates are corroborated by the gas phase experiments of Dowling ${ }^{16}$ as well as the measurements in water by Buch and $\mathrm{Dahm}^{24}$ and suggest that the smallest diffusion scales may have been resolved in these experiments, at least at the lower Reynolds numbers.

As mentioned previously, earlier measurements were also made using a 512 pixel linear photodiode array, yielding line images versus time (two-dimensional streak data). The images were recorded at Reynolds numbers of 1000,2000 , and 3000 , and were centered on the jet axis, spanning about a tenth of the local jet diameter (at $x / d=300$ ). The local Kolmogorov scale was resolved in each case. These data were analyzed in several ways. First, the individual time records of each of the 512 pixels of the array were processed using the box-counting algorithm discussed previously. The results matched those of the single-point data. Next, the 512 element line images were processed separately, providing us with spatial results without invoking Taylor's hypothesis. Once again, while the dynamic range of the data is limited by the number of pixels in the array, we find a similar behavior (Fig. 6).

In addition to the one-dimensional box-counting algorithm, a two-dimensional tiling program was also employed on the streak image data. For those data, the data acquisition rate of the linear array was adjusted to be proportional to the local flow velocity, providing a two-dimensional streak image. This minimized changes in the stretching between the spatial and temporal dimensions with changes in Reynolds number. The results are shown in Fig. 7. Note that since this result uses two-dimensional tiling, $D(\lambda)$ is now bounded by the limiting values of 1 and 2. Qualitatively, the results from the line images and the full streak images are similar. Nevertheless, there is a small and systematic quantitative differ-

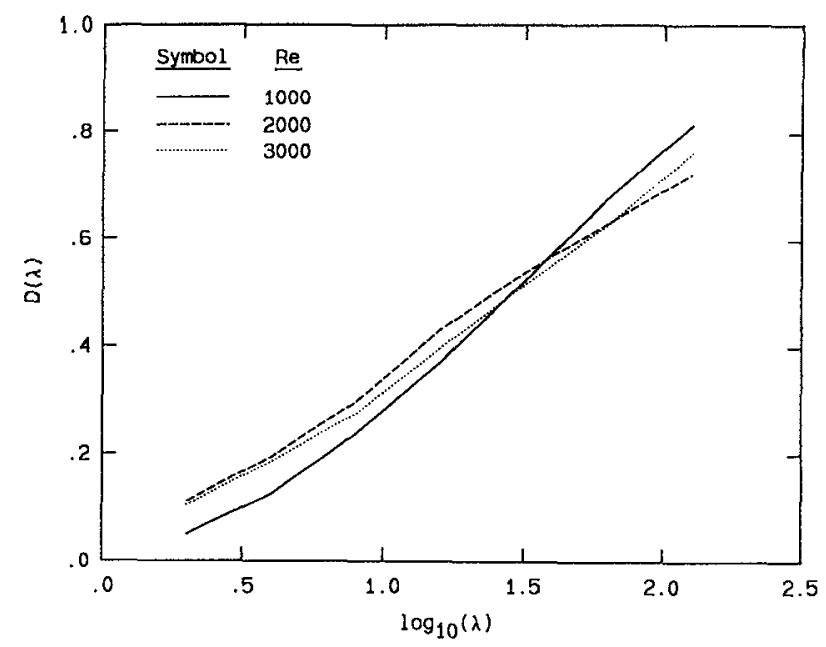

FIG. 6. Line image results.

ence-the slopes of the higher-dimensional data are steeper. We believe that this difference is not an artifact.

Finally, single-point measurements were made off the jet centerline at $\eta=r / x$ of 0.06 and 0.13 , at a Reynolds number of 8600 . These results are shown in Fig. 8, with a corresponding centerline curve. The similarity in the three results is noteworthy.

This ensemble of on- and off-axis, one- and two-dimensional measurements is significant because it serves to examine the effect of analyzing temporal, rather than spatial, data. If one wishes to describe a spatial scalar interface, rather than an Eulerian point concentration history, some type of assumption is required, since we have insufficient information about the velocity field to make an exact conversion. We have used the calculated mean centerline velocity to convert the temporal data to spatial results in Fig. 5. Near the jet centerline, we feel this is acceptable, while near the edge of the jet, we recognize that the errors involved may not be negligible. Rather than open the issue of how accurate this

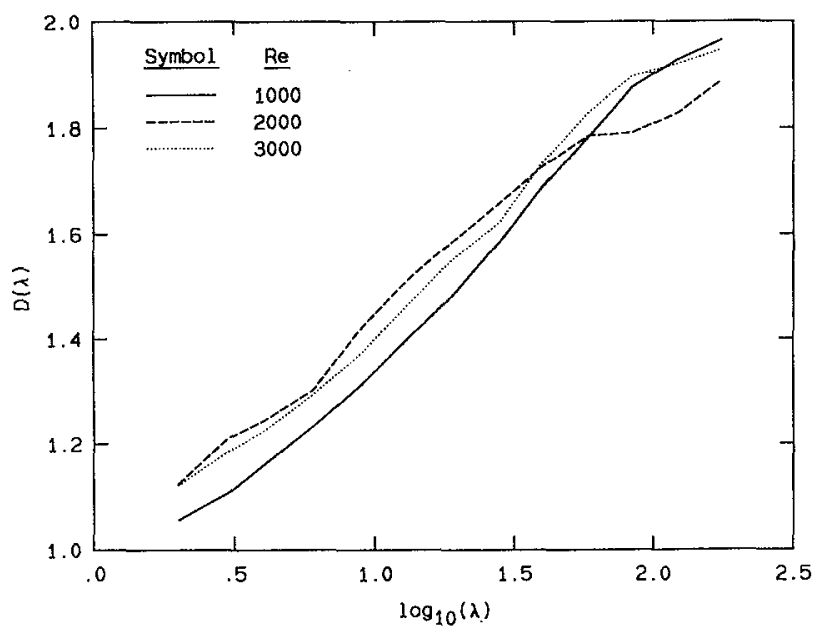

FIG. 7. Two-dimensional (streak image) results. 


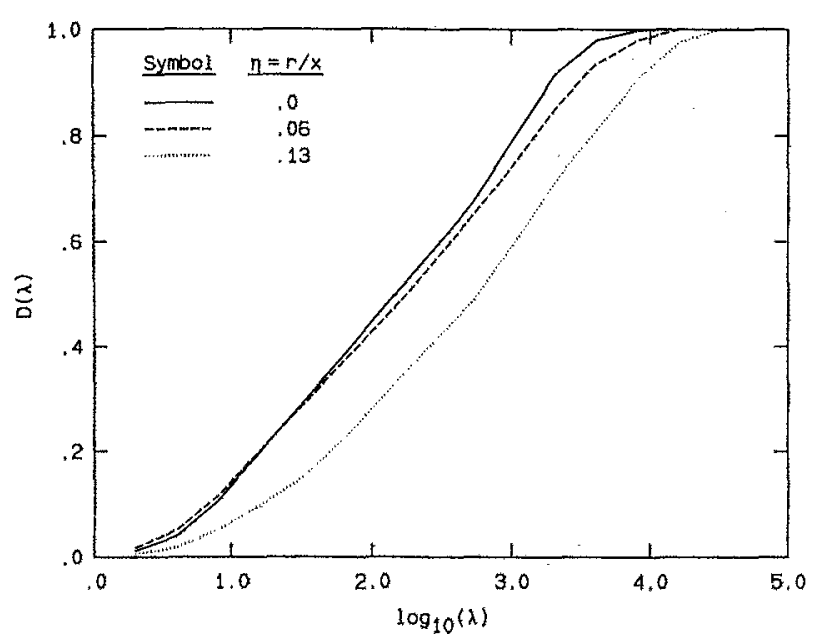

FIG. 8. Off-axis single-point results $(\operatorname{Re}=8600, \eta=r / x)$.

implementation of Taylor's hypothesis may be, we note $a$ posteriori that the results are insensitive to its use. The behavior is qualitatively the same for the single-point measurements in Fig. 5, the off-axis results in Fig. 8, which could exhibit the greatest variance from Taylor's hypothesis, and the results in Fig. 6 from true (line image data). The latter, of course, do not utilize Taylor's hypothesis at all. The twodimensional tiling algorithm and the corresponding results in Fig. 7 again demonstrate similar behavior.

It has been suggested (Sreenivasan and Meneveau ${ }^{7}$ ) that if long records are used in the box-counting algorithm, they may mask local power-law fractal behavior. We do not find this to be the case. This issue is discussed in the Appendix.

The persistence of these results may be traceable to a strong stochastic character of the interfaces. In an effort to model this behavior, we devised a simple Monte Carlo description of the threshold crossings. A program was written to produce a record of crossings that were randomly spaced with a lognormal distribution. PDF's of our measured crossing spacings independently show that the lognormal distribution is a good approximation. Two parameters, the location of the maximum and the width of the distribution, were allowed. Figure 9 includes the result for a Reynolds number

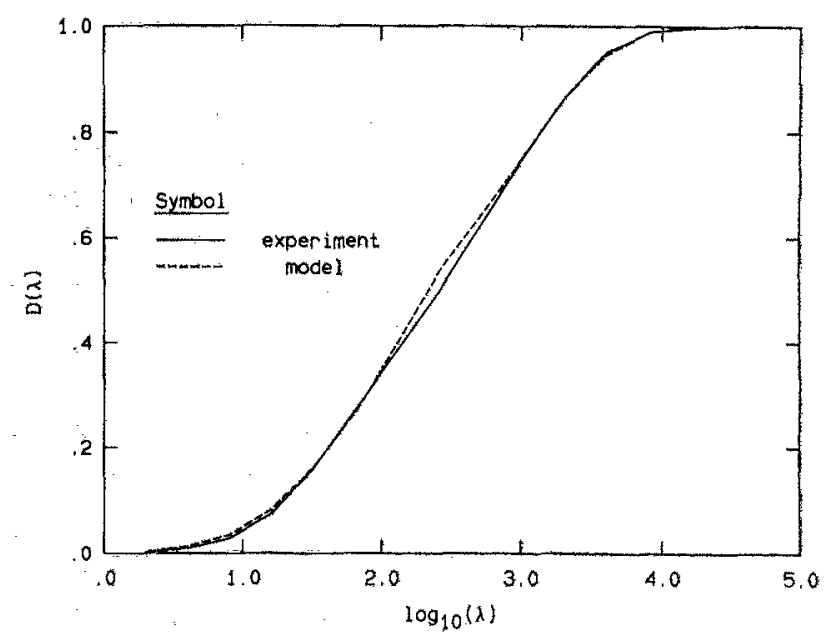

FIG. 9. Comparison of lognormal model and experiment $(\operatorname{Re}=5800$ ).

of 5800. The agreement between model and experiment is good.

Finally, we wish to address the important issue of the choice of the value of the threshold. Recall that in the boxcounting algorithm, a specific concentration value is chosen, and crossings of this threshold are located. These crossings correspond to the passage of an isoconcentration surface possessing the threshold value. For the results presented previously, the local mean concentration $\bar{c}$ was used as the threshold value. Recall that similar behavior is observed both on and off axis, despite the fact that the local (absolute) mean concentration changes significantly with $\eta=r / x$.

We examined the entire range of possible thresholds for our single-point data, both on axis and off axis at $\eta=0.13$. Three-dimensional plots of $D(\lambda)$ versus threshold and $\log (\lambda)$ are shown in Figs. 10 and 11. A contour plot of the on-axis data offers ant alternate representation of the results in Fig. 10 (Fig. 12). As can be seen, the continuous variation of the $D(\lambda)$ curves with $\lambda$ persists for a rather large range of threshold values on either side of the local mean concentration. As the threshold is either increased or decreased, two effects are observed. The sloping region shifts position, achieving the asymptotic value of 1 at progressively larger scales, and a bump appears at smaller values of $\lambda$. While the

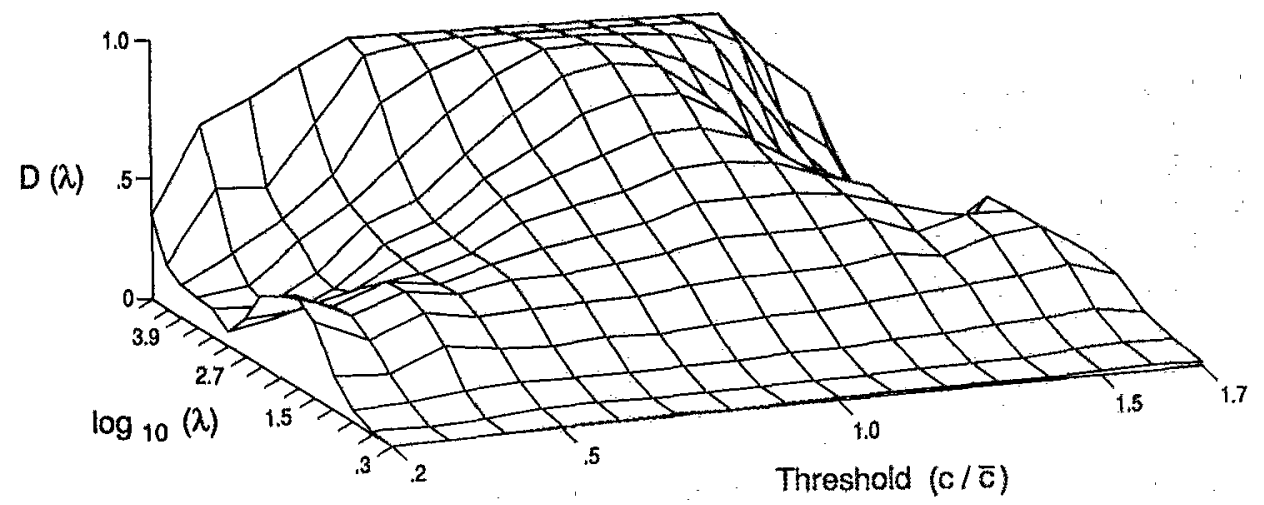

FIG. 10. Three-dimensional plot of $D(\lambda)$ versus threshold and $\lambda$ for singlepoint, on-axis measurements $(\operatorname{Re} \approx 10000)$. 


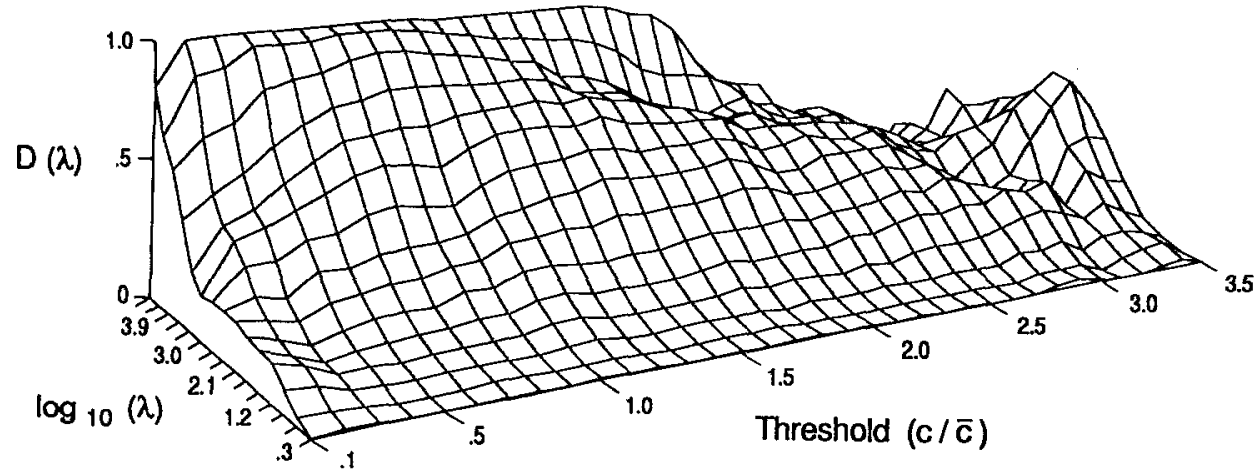

FIG. 11. Three-dimensional plot of $D(\lambda)$ versus threshold and $\lambda$ for singlepoint, off-axis measurements $(\operatorname{Re} \approx 10000, \eta=0.13$ ). linear scale may not make it clear, the off-axis data exhibit a similar bump at small scales as the on-axis data, when viewed at smaller values in logarithmic coordinates.

To investigate whether this behavior was a consequence of the random character of the concentration signal rather than the fluid dynamics of the turbulent jet, we processed data of laser light scattered from a very dilute, constant concentration solution. These data are essentially measurements of (shot) noise, possessing a white power spectrum. A narrow Gaussian filter was used as a cutoff to eliminate the highest frequencies. The corresponding three-dimensional plot of $D(\lambda)$ for these noise data is displayed as Fig. 13. The global behavior is very similar to Fig. 10, even though, for the noise data, no fluid mechanics are involved.

Note that, despite the qualitative similarities, there are several important quantitative differences between Fig. 10 and Fig. 13. The rise to the asymptotic value of 1 has a different slope in the two plots. This slope is a measure of the width of the distribution of scales. The scale at which the midpoint

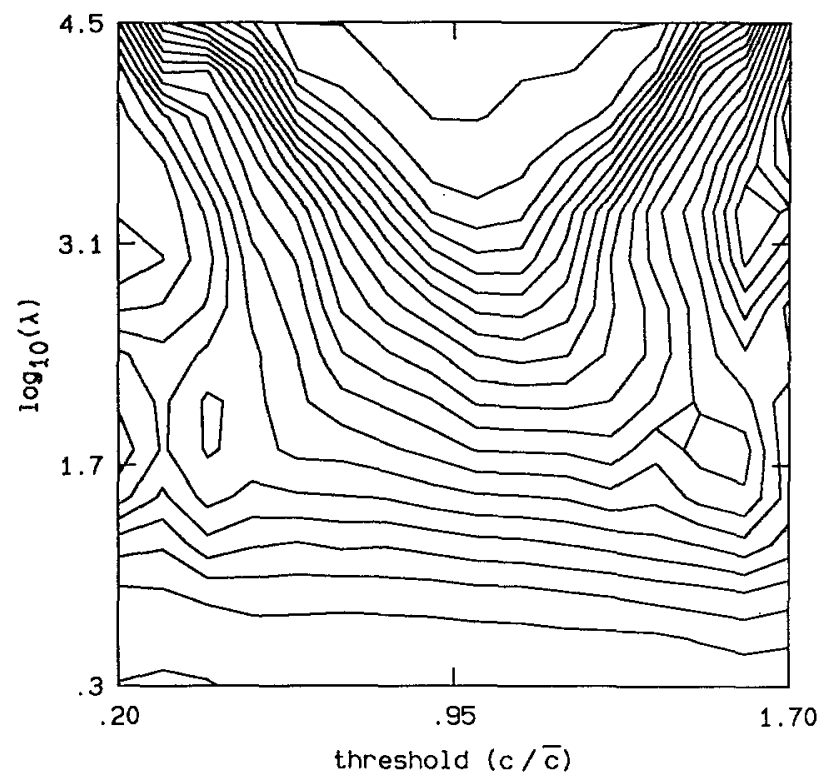

FIG. 12. Contour plot of data in Fig. 10. of the rise is found, which relates to the location of the maximum of the distribution, also varies. Finally, the range of normalized concentrations is much narrower in Fig. 13 than in Fig. 10, reflecting the very different transition PDF's in each case. While there is no particular relationship between these quantities for the noise data and the jet results, we do expect that they will provide useful information in the comparison of fluid flows. This topic, which is somewhat beyond the intent of the present paper, is left for future discussions.

To further understand the three-dimensional plots, we need to consider the nature of the concentration signal we are examining. The possible scalar values are bounded by zero and some upper limit, and the measured concentration time history exhibits many maxima and minima. Near the mean, relatively few of these extrema are encountered. On the other hand, for thresholds approaching the highest or lowest values detected, many such turning points are found, and they may dominate the statistics. Picture a local minimum in concentration, with a roughly parabolic dependence of $c(t)$ in its vicinity. Imagine then a threshold level that is slowly decreased toward the minimum value. Two threshold crossings are encountered, which, as the threshold is lowered, move closer together. These eventually (almost) join before the threshold drops below the minimum(recall that the signal is discretely sampled in time). Thus near the turning points there can be a separation of scales; one length is associated with the typical distance between extrema and the other is a much smaller scale associated with the spacing of crossings within pairs at each extremum.

This scale separation manifests itself in the $D(\lambda)$ plots in two primary ways. One is the appearance of a bump at the smallest scales, traceable to the double crossings at each extremum. This bump is indicative of a characteristic length at that scale. The second is that the rise of $D(\lambda)$ to its asymptotic value of unity is shifted to larger scales as the threshold is increased, or decreased, from the local mean. This is ascribable to what could be called conservation of spacing. Pairs of crossings drop out as the threshold moves past their extrema, producing a larger length scale associated with the distance between crossings to either side of the pair. This is enhanced by crossings within a pair coming closer together as the threshold approaches their extremum, causing the spaces between adjacent pairs to correspondingly increase. 


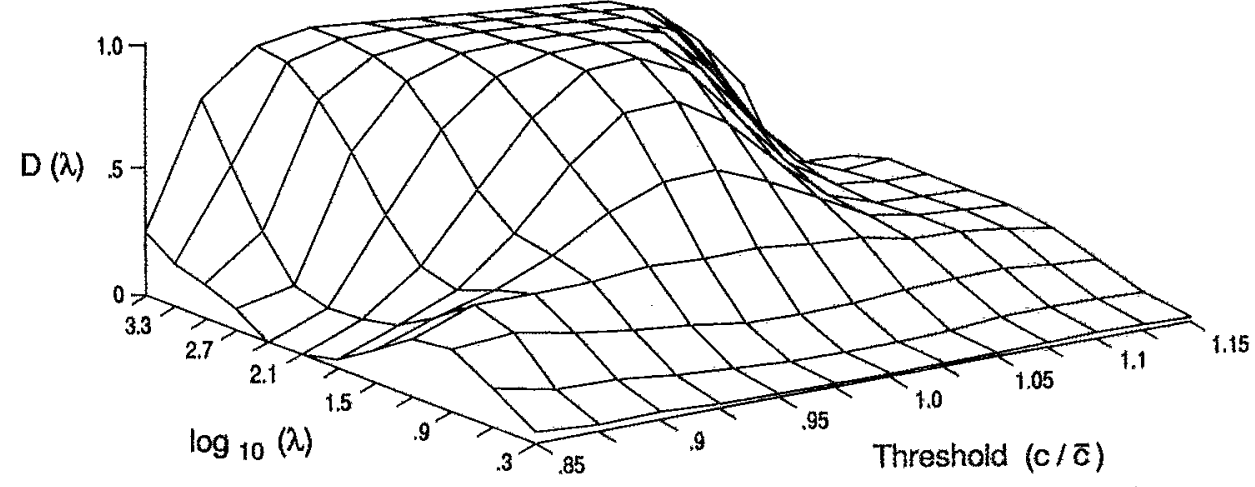

FIG. 13. Three-dimensional plot of $D(\lambda)$ for noise data.
Both the bump at small scales and the shift in the rise are evident in all of the three-dimensional $D(\lambda)$ plots.

\section{CONCLUSIONS}

In this paper, we have discussed high resolution scalar field measurements conducted in the far field of a turbulent jet. We have examined single-point measurements, one-dimensional line images, two-dimensional streak images, ensembles of long records and short records, of on- and off-axis data in jet flows spanning over a decade in Reynolds number. We find no evidence that isoscalar surfaces are described by a fractal (power-law) similarity.

There have been a number of papers documenting experimental measurements of fractal dimension in turbulent flows, e.g., Sreenivasan and Meneveau, ${ }^{7}$ Sreenivasan et al., ${ }^{6}$ Meneveau, ${ }^{25}$ Prasad and Sreenivasan, ${ }^{8}$ and Prasad and Sreenivasan, ${ }^{9}$ among others. These authors have concluded that fractal (power-law) similarity is applicable to the description of scalar interfaces in a wide variety of turbulent flows, including jets.

We should state explicitly that our results are in direct disagreement with a significant portion of the previously cited papers. While those authors have increasingly cautioned against the use of one-dimensional data to search for fractal behavior, they have, at the same time, offered evidence in support of agreement between single-point measurements, line images, and two-dimensional images. Our examinations in this paper include the first two of the three methods, and do not support the notion of a constant fractal dimension. Instead, we found that, over a wide range of locations, thresholds, flow conditions, and different types of measurements, the behavior is well modeled by lognormal statistics.

We offer one possibility how previous researchers concluded that a constant fractal dimension is appropriate where we do not. As we have demonstrated previously, the influence of pairs of transitions near extrema may cause a bump or even a fairly level region in the $D(\lambda)$ curves at particular selections of the threshold. The thresholds used by Sreenivasan et al. are much lower than the local mean and may fall in such a region. It could be that the bump feature, particularly when finding the dimension by fitting a line to the log-log plots, gave the appearance of a constant dimension. It was only the direct differentiation of the curves that allowed us to generate plots such as Fig. 10, thereby discerning the bump.

Additionally, there is an issue concerning the two-dimensional results of Sreenivasan et al. and our findings. We have not analyzed two-dimensional spatial images at this time. Our use of two-dimensional streak images generated by the linear array, an approximation of spatial images, yielded results similar to those of our other data. However, we recognize the analysis of these data involves several assumptions which preclude exact comparison, and therefore we cannot directly confirm or deny the two-dimensional results.

Our data and analysis force us to conclude that, at least in the far field of turbulent jets, scalar interface geometry cannot be described in terms of a constant fractal dimension, under any of the flow conditions we have examined in our experiments. Further, we have found that the behavior of isoscalar surfaces with the local mean value agrees well with a simple, lognormal, stochastic model. For values substantially different than the local mean, similar behavior is observed if the boundedness of the signal is taken into account. This suggests to us that the notion of constant fractal dimension, if applicable to the jet scalar field at all, is something of a special case rather than the norm. These conclusions were the reason for avoiding the word "fractal" in our title, as that was originally coined by Mandelbrot to denote the power law of Eq. (1). In fact, Feder credits Mandelbrot as having retracted his original definition of a fractal. Mandelbrot's revised and more relaxed definition is not tied to a power law (Hausdorff-Besicovitch) dimension, but rather to a general geometric property of similarity (Feder, ${ }^{26} \mathrm{p} .11$ ). We appreciate that the flow in the far feld of turbulent jets is characterized by a host of similarity properties, indeed spanning the full spectrum of scales (Dowting ${ }^{16}$ ). We note, however, that fractal (power law) behavior is not compelled by similarity.

This conclusion suggests that perhaps the premise for a constant (power-law) fractal dimension at moderate to high Reynolds numbers should be reexamined, i.e., the proposal that 


$$
N(\lambda) \propto \lambda^{-D}
$$

can serve as the measure of the number of elements of scale $\lambda$ required to cover the scalar interface, where $D$ is a constant. In particular, we recognize that Eq. (1) is not dimensionally correct, at least as it stands; only a dimensionless group can be related to a pure number. Equation (1) could be made dimensionally correct using either inner variable scaling, i.e.,

$$
N(\lambda) \propto(\lambda / \lambda,)^{-D},
$$

where $\lambda$, is the scalar diffusion scale, or outer scaling, i.e.,

$$
N(\lambda) \propto(\lambda / \delta)^{-D},
$$

where $\delta$ is the local jet diameter, for example. There are difficulties with such proposals, however, especially for values of the fractal dimension $D$ substantially different from the corresponding Euclidean values.

At least in the inertial range where one might expect the power-law fractal similarity to hold, it would imply either that the inner (Kolmogorov/Batchelor) scales [Eq. (7a)], or the outer scales [Eq. (7b)] are imposed on the dynamics. Yet, the high Reynolds number dimensional analysis and similarity scaling derived from the early Kolmogorov ${ }^{27}$ hypotheses, which yield the $-\frac{5}{3}$ spectrum power laws, is equivalent with the assumption, among other things, that the only variables that enter in the inertial range cascade dynamics are the local scale $\lambda$ (or wave number $k$ ) and the kinetic energy dissipation rate $\epsilon$. The scaling laws implied by Eqs. (7a) and (7b) are inconsistent with this assumption, as no other length scale is derivable from $\lambda$ and $\epsilon$. While additional length scales are admissible in the subsequent turbulence similarity hypotheses that incorporate the intermittency corrections (e.g., Kolmogorov, ${ }^{28}$ Oboukhov, ${ }^{29}$ and Gurvich and Yaglom ${ }^{20}$ ), these enter through similarity laws that are much weaker than power laws, e.g., lognormal, and provide only small corrections to the originally proposed - $\{$ spectrum power-law exponent. Alternatively, the original Kolmogorov similarity proposals of a scaleless cascade range are found to be very nearly correct (also see the related discussion in Dimotakis, ${ }^{30}$ p. 482). In contrast, Eqs. (7) are equivalent to a strong dependence of the dynamics on outer, or inner, scales. Consequently, it would appear that a powerlaw description of the statistics at the high Reynolds numbers of interest is inappropriate, not only on the basis of the experimental data we have presented, but also on the basis of dimensional analysis and similarity arguments.

\section{ACKNOWLEDGMENTS}

We would like to acknowledge the assistance of $D$. Dowling and D. Lang with the experiments, helpful suggestions from W. Ashurst, as well as discussions with G. Losi. One of us (PD) would also like to acknowledge several conversations with $B$. Mandelbrot on the occasion of his visits at Caltech over the last ten years, or so, as well as with $\mathrm{K}$. Sreenivasan during his stay at Caltech as a Visiting Professor a few years ago.

This work was performed under AFOSR Grants No.
83-0213 and No. 88-0155, and GRI Contract No. 5087-2601467.

\section{APPENDIX: ASPECTS OF THE FRACTAL ANALYSIS}

This appendix expands upon several topics that we feel deserve a more detailed discussion, including aspects of the box-counting algorithm, the treatment of noise in the data, and the consequence of analyzing shorter data records.

There are subtle end effects that can occur with the boxcounting algorithm. If the record submitted for box counting is a nonintegral number of a particular tile size in length, the last tile extends past the end of the record. Some type of weighting scheme for this fractional end tile may suggest itself, but such weighting requires the essentially $a d$ hoc assignment of the corresponding probability of a transition. We have conducted numerical experiments utilizing the onedimensional Cantor set, using nonintegral numbers of tiles. They displayed sawtooth oscillations that varied in wavelength as a function of tile size, consistent with end effects. We verified that various weighting schemes do not correct the difficulty. As a result, we decided that tile sizes should be exact factors of the total record length. This ensures that there is always an integral number of tiles in the record, and thus eliminates end effects of this type.

It is significant to note that, using only integral tiles, the $\log [N(\lambda)]$ curves must be nonincreasing with increasing tile size. It cannot require more larger tiles than smaller ones to cover the same transitions. Note, also, that local increases in the $\log [N(\lambda)]$ curve would yield a negative fractal dimension for the corresponding range of scales. This is, of course, inadmissible. As an example, imagine a record six elements long, in which the third and fourth elements contain transitions Fig. 14(a). If tiles of size 2 are placed on the record, the central tile will cover both transitions, and $N(\lambda)$ is 1. If two tiles of size 3 are laid down, then both the first tile, covering elements one through three, and the second tile, covering elements four through six, will cover transitions. In that case, $N(\lambda)$ will be 2 . This example may appear to contradict the statement that the $\log [N(\lambda)]$ curves must be

(a)

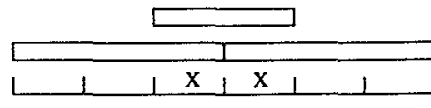

$N(2)=1.0$

$\mathrm{N}(3)=2.0$

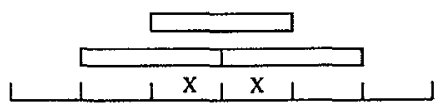

$\mathrm{N}(2)=1.50$

(b)

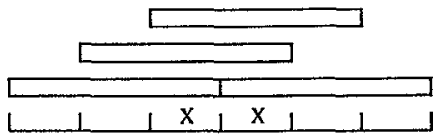

$\mathrm{N}(3)=1.33$

FIG. 14. Dependence on tile starting location. Tiles covering transitions, denoted by " $X$," are indicated by narrow rectangles . (a) Fixed starting location. (b) Weighted average over possible starting locations. 
nonincreasing with tile size. It actually illustrates another issue.

We note that there is no preferred starting location or reference point when tiling a record. The apparent difficulty in the example is a consequence of the particular choice of starting location. If the tiling is done as many times as there are possible starting locations, all the information available is extracted. In the example, there are two possible starting locations for tiles of size 2 , either on even or odd elements, and three for tiles of size 3 [Fig. 14(b) ]. If the results with all possible starting locations are ensemble averaged, we find that $N(\lambda)$ is 1.5 for tiles of size 2 , and 1.33 for tiles of size 3 , The need to shift tile locations and the restriction of having an integral number of tiles in each record suggested the choice of tile sizes and record lengths that are powers of 2 of the sampling unit. We have found that using just eight different starting locations, equally spaced, does a reasonable job of reducing the effect illustrated in Fig. 14.

In addition to these end-effect difficulties, there is the issue of the influence of unavoidable noise in the data. It has been appreciated for some time that the presence of noise in a signal can profoundly affect the statistics of level crossings (e.g., Rice ${ }^{31,32}$ ). Since the box-counting algorithm utilizes level crossings or transitions, the issue of noise is important. To illustrate this, Fig. 15 displays a small segment of an unfiltered signal and the corresponding optimally (Wiener-) filtered signal. Bear in mind that the unfiltered signal appears as noisy as it does because it has been oversampled, as dictated for an effective implementation of the Wiener filter. It is apparent that noise grossly affects the crossings of the threshold (mean level), and that a proper treatment of this aspect of the data is an essential component of the signal and data processing. Note that noise can cause crossings where the signal approaches the threshold but does not cross $i t$. By not only adding crossings in the vicinity of a true crossing, but also where there was no true crossing nearby, the noise contaminates the results at scales much larger than might be suspected, as well as at smaller scales.

The effect illustrated in Fig. 15 is accentuated because the signal is oversampled. Nevertheless, even a small addi-

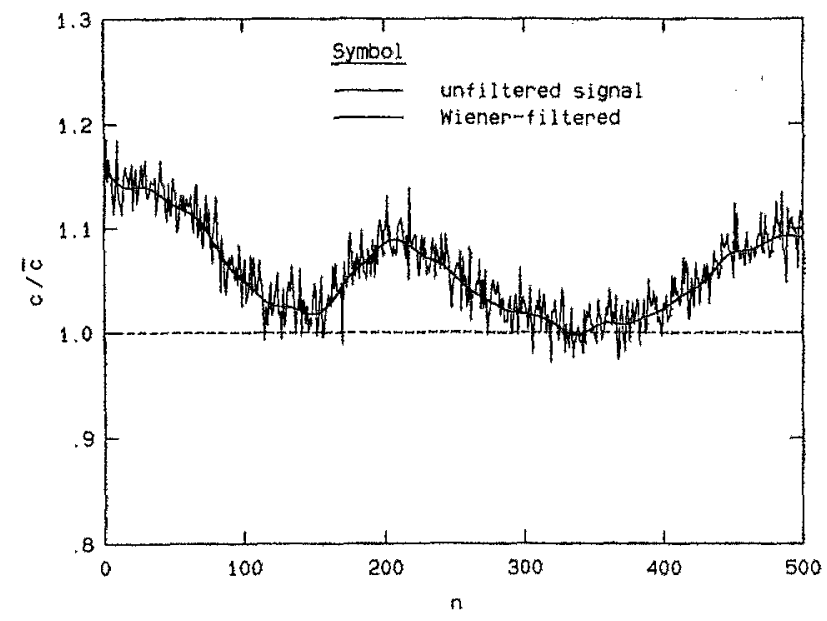

FIG. 15. Effect of noise on transitions ( $R e=2940$ ).

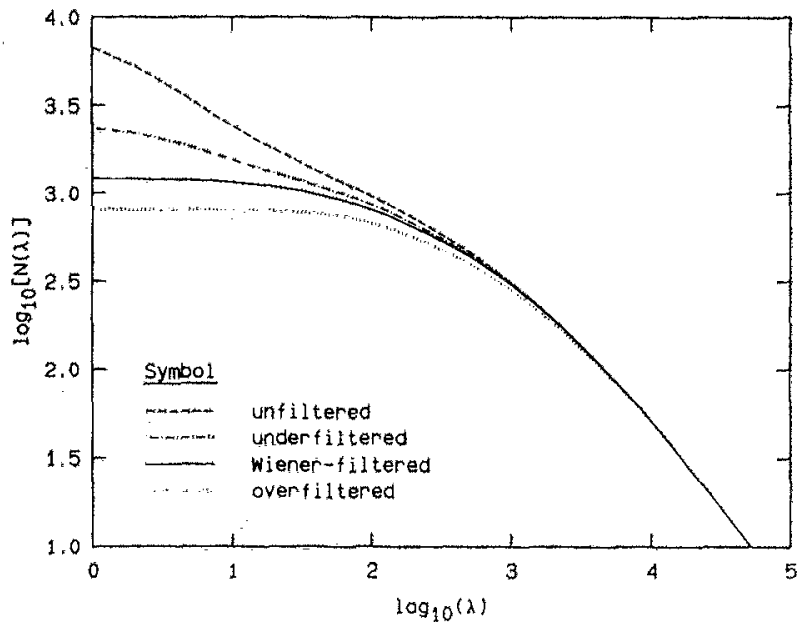

FIG. 16. Infiuence of noise on $\log [N(\lambda)]$ curves $(\mathrm{Re}=2940)$.

tion of noise at the smallest scales can influence the results significantly. As a demonstration of this behavior, a data record from a particular run was processed unfiltered, with a variety of Gaussian filters of differing widths, as well as with the optimal (Wiener) filter. The Gaussian filter, if chosen with an appropriate width (which, however, is not known $a$ priori), may serve as an approximation to the Wiener filter. Figure 16 shows the results for no filtering, insufficient filtering, optimal filtering, and overfiltering. It is evident that the presence of noise in the data may result in a region of nearly constant slope on the $\log [N(\lambda)]$ plots, which may even be interpreted as two regions of somewhat constant slope. In fact, the $D(\lambda)$ plots computed from these curves exhibit a "bump" at small scales, as previously discussed in the text. For these data, the slope at smaller scales is steeper than at larger scales, and there is a slight roll-off at the smallest values. When the noise is removed (the Wiener-filtered case), however, these constant slopes do not persist.

Two additional points are illustrated by Fig. 16. First, as noted above, the contamination of the results is not limited to the small scales or high frequencies where the noise dominates. It extends to much larger scales. The various curves do not coincide until a scale of at least 500 or 1000 sampling units. This is larger, by a factor of $20-30$, than the ratio of the signal spectrum intercept with the noise floor to the sampling frequency. We are forced to conclude that the influence of noise, if permitted, may encroach into scales that are well resolved with respect to space, time, and SNR. In short, a large SNR is not sufficient to ensure that noise has not affected the results. Additional care, such as use of the Wiener filter, is typically necessary. Second, it is evident from Fig. 16 that either too wide or too narrow a filter kernel can also affect the results. Filtering only the highest frequencies out of the noise is insufficient, yielding a similar rise in $N(\lambda)$ at the smallest scales as the unfiltered data. The overfiltered result, although similar in shape to the correct curve, has also been compromised. Thus very careful consideration must be given to the handling of noise in level crossing data that are analyzed for fractal behavior.

A final issue that should be assessed is the consequence 


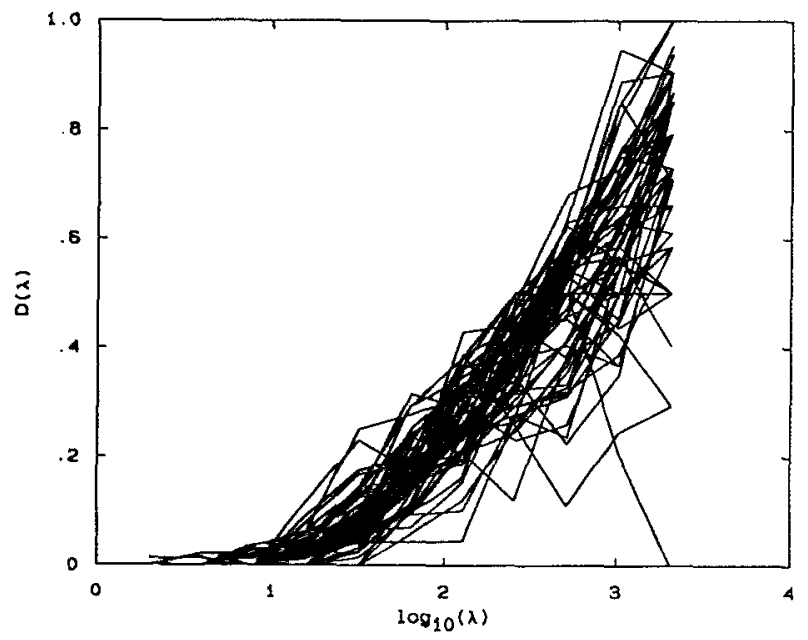

FIG. 17. Many individual short records $(\operatorname{Re}=2940$ ).

of analyzing shorter data records with the box-counting algorithm, as was mentioned in the text. Shorter records have been suggested ( $c f$. Sreenivasan and Meneveau ${ }^{7}$ ) because of the possibility that scalar interfaces might display a particular power-law fractal behavior on a local length or time scale, but that over larger scales, fluctuations or variations in the local properties could smear it out. Our record lengths varied in length from about 8-70 large scale times. Such a number of large scales provided good statistics. We saw no indication the record lengths were influencing the results.

Nevertheless, we examined the behavior of shorter record lengths. Rather than ensemble averaging either the $\log [N(\lambda)]$ plots or the $D(\lambda)$ curves for many short records, we superimpose many separate realizations on one plot (Fig. 17). The results shown are for a Reynolds number of 2940. The individual curves do not exhibit a flat (horizontal) region, which would imply a constant fractal dimension. Additionally, the ensemble average, easily constructed by eye, resembles the result displayed in Fig. 5. Even shorter records were analyzed, for the entire range of Reynolds numbers, both on and off axis, and the outcome was the same for re- cord lengths of all sizes. We conclude that record length is not a factor in our results.

'B. B. Mandelbrot, J. Fluid Mech. 72, 401 (1975).

${ }^{2}$ B. B. Mandelbrot, Fractals: Form, Chance, and Dimension (Freeman, San Francisco, 1977).

${ }^{3}$ B. B. Mandelbrot, Fractals and Turbulence: Attractors and Dispersion, Lecture Notes in Mathematics (Springer, Berlin, 1977), Vol. 615, pp. 8393.

${ }^{4}$ B. B. Mandelbrot, The Fractal Geometry of Nature (Freeman, San Francisco, 1983).

${ }^{5}$ F. C. Gouldin, AIAA J. 26, 1405 (1988).

${ }^{6} \mathrm{~K}$. R. Sreenivasan, R. Ramshankar, and C. Meneveau, Proc. R. Soc. London Ser. A 421, 79 (1989).

${ }^{7}$ K. R. Sreenivasan, and C. Meneveau, J. Fluid Mech. 173, 357 (1986).

${ }^{8}$ R. R. Prasad and K. R. Sreenivasan, Exp. Fluids 7, 259 (1989).

${ }^{9}$ R. R. Prasad and K. R. Sreenivasan, Phys. Fluids A 2, 792 (1990).

${ }^{10}$ P. E. Dimotakis, R. C. Miake-Lye, and D. A. Papantoniou, Phys. Fluids 26, 3185 (1983).

"W. J. A. Dahm, Ph.D. thesis, California Institute of Technology, 1985.

${ }^{12} \mathrm{~S}$. Green and G. Losi, California Institute of Technology, Ae104c Laboratory Report (unpublished).

${ }^{13}$ W. J. A. Dahm and P. E. Dimotakis, AIAA J. 25, 1216 (1987).

${ }^{14}$ N. Wiener, Extrapolation, Interpolation and Smoothing of Stationary Time Series (Wiley, New York, 1949).

${ }^{15}$ W. H. Press, B. P. Flannery, A. A. Teukolsky, and W. T. Vetterling, $N u$ merical Recipes. The Art of Scientific Computing (Cambridge U.P., Cambridge, 1986).

${ }^{16}$ D. R. Dowling, Ph.D. thesis, California Institute of Technology, 1988.

${ }^{17}$ D. R. Dowling, D. B. Lang, and P. E. Dimotakis, Exp. Fluids 7, 435 (1988).

${ }^{18}$ C. J. Chen and W. Rodi, Vertical Turbulent Buoyant Jets: A Review of Experimental Data (Pergamon, New York, 1980).

${ }^{19}$ L. A. Smith, J-D. Fournier, and E. A. Spiegel, Phys. Lett. A 114, 465 (1986).

${ }^{20}$ A. S. Gurvich and A. M. Yaglom, Phys. Fluids, 10, Suppl., 59 (1967).

${ }^{21}$ C. A. Friehe, C. W. Van Atta, and C. H. Gibson, AGARD Turbulent Shear Flows Report No. CP-93, 1971, pp. 18.1-18.7.

${ }^{22}$ D. R. Chapman, AIAA J. 17, 1293 (1979).

${ }^{23}$ G. K. Batchelor, J. Fluid Mech. 5, 113 (1959).

${ }^{24}$ K. A. Buch and W. J. A. Dahm, Bull. Am. Phys. Soc. 34, 2264 (1989).

${ }^{25} \mathrm{C}$. Meneveau, Ph.D. thesis, Yale University, 1989.

${ }^{26} \mathrm{~J}$. Feder, Fractals (Plenum, New York, 1988).

${ }^{27}$ A. N. Kolmogorov, Dokl. Akad. Nauk SSSR 30, 299 (1941); reprinted in Usp. Fiz. Nauk 93, 476 (1967) [Sov. Phys. Usp. 10, 734 (1968)].

${ }^{28}$ A. N. Kolmogorov, J. Fluid Mech. 13, 82 (1962).

${ }^{29}$ A. M. Oboukhov, J. Fluid Mech. 13, 77 (1962).

${ }^{30}$ P. E. Dimotakis, in Turbulent Reactive Flows, Lecture Notes in Engineering (Springer, Berlin, 1989), Vol. 4, pp. 417-485.

${ }^{31}$ S. O. Rice, Bell Syst. Tech. J. 23, 282 (1944).

${ }^{32}$ S. O. Rice, Bell Syst. Tech. J. 24, 46 (1945). 